\title{
Optimización del tiempo de proceso en el reacondicionamiento de pozos en un campo de la Cuenca Oriente ecuatoriana aplicando herramientas de mejoramiento continuo
}

\section{Optimization of process time in the well reconditioning in a basin field Ecuadorian east applying improvement tools continous}

ARTÍCULO ORIGINAL

Recibido: 03/09/2019

Aprobado: 29/11/2019

\author{
Ruiz Pozo Renán \\ (1) https://orcid.org/0000-001-8788-6227 \\ Petroamazonas \\ renan_ruiz@petroamazonas.gob.ec
}

\author{
Gallegos Eras Álvaro \\ () https://orcid.org/0000-00034049-858X \\ Escuela Politécnica Nacional. \\ alvaro.gallegos@epn.edu.ec
}

\section{RESUMEN}

El reacondicionamiento de pozos es uno de los procesos más importantes en la industria petrolera, pues permite recuperar o incrementar la producción hidrocarburífera de un pozo petrolero que por condiciones mecánicas o del reservorio, se encuentra produciendo volúmenes menores a su valor óptimo. El reacondicionamiento de pozos de la empresa petrolera estatal Petroamazonas EP en un campo de la Cuenca Oriente ecuatoriana fue analizado con el fin de definir una propuesta de mejoramiento utilizando herramientas de común uso en la Ingeniería industrial. Considerando el punto de vista de los clientes del proceso, se determinó que el parámetro sujeto a mejoramiento es el tiempo. Para determinar las operaciones a ser optimizadas, se recopiló y analizó la información disponible de los reacondicionamientos realizados en el campo petrolero estudiado, desde enero 2015 a enero 2017. Utilizando análisis de Pareto se determinaron dos operaciones a ser sometidas a mejoramiento: Inicialización y Completación. Para estas operaciones se realizaron gráficas de control con el fin de determinar valores fuera de los rangos de tiempo esperados. A los puntos más alejados se les aplicó un análisis causa-efecto con el fin de determinar la causa principal de la falla. Considerando los resultados del análisis, se elaboró una propuesta de mejoramiento del proceso de reacondicionamiento de pozos.

Palabras clave:

Gráficas de control; mejoramiento continuo; proceso de optimización; reacondicionamiento de pozos.

\begin{abstract}
Workover of wells is one of the most important processes in the oil industry because it recovers or increases the hydrocarbon production of an oil well that, because of mechanical conditions or the reservoir, is producing volumes less than their optimal value. The workover process of the national oil company Petroamazonas EP in an oilfield in the Ecuadorian Oriente Basin was analyzed in order to define an improvement proposal using tools commonly used in Industrial Engineering. Considering the point of view of the clients of the process, it was determined that the parameter subject to improvement is the time. In order to determine the operations to be optimized, the available information of the workovers made in the oilfield, from January 2015 to January 2017, was collected and analyzed. Using Pareto analysis, two operations to be improved were determined: Initialization and Completion. For these operations, control charts were performed in order to determine values outside the expected ranges of time. At the farthest points a cause-and-effect analysis was applied to determine the main cause of the failure. Considering the results of the analysis, a proposal was developed to improve the workover process.
\end{abstract}

\section{Keywords:}

Control graphics; continuous improvement; optimization process; workover.

\section{INTRODUCCIÓN}

En los meses de enero y febrero del año 2016 los precios del petróleo alcanzaron records de valores bajos, que no se habían visto desde el año 2003 
(OPEC, 2016, p. 5). La baja de los precios del petróleo que inició en agosto de 2014 puso en primer plano la necesidad de optimizar procesos y reducir los costos operativos de las empresas petroleras a nivel mundial.

A partir del año 2015 se hizo evidente que los precios del petróleo tardarán en recuperarse. En este contexto, adquiere importancia la aplicación de herramientas de mejoramiento continuo, puesto que permiten mejorar las características del producto o servicio, el ahorro de costos por disminución de fallas y la obtención de menores tiempos de ciclo de los procesos (Knowles, 2011, pp. 14-23).

La empresa petrolera estatal ecuatoriana Petroamazonas EP no es ajena a este escenario de precios bajos y restricciones presupuestarias, por lo tanto, ha emprendido acciones tendientes a la reducción de los costos en cada una de sus áreas operativas.

En el año 1982, Edwards Deming publicó un libro cuyo título original en inglés es "Out of the crisis" y su título en español es "Calidad, productividad y competitividad: la salida de la crisis" el cual ha tenido varias ediciones por su incidencia en el mejoramiento de los procesos productivos. El mensaje que deja este libro es que la productividad no tiene que reñirse con la calidad, y que el compromiso con la calidad es el cambio al cual deben orientarse las empresas (Deming, 1986, p. 1). Este mensaje tiene aplicación en los momentos actuales, a pesar de haber sido escrito hace más de 37 años.

El proceso de reacondicionamiento de pozos es parte del macroproceso de producción de petróleo, y consiste en utilizar una torre de reacondicionamiento para realizar trabajos específicos dentro de un pozo petrolero, tales como: cambiar la zona productora, disparar zonas para conectar el reservorio con la tubería de producción, solucionar problemas mecánicos, etc.

Desde enero 2015 a enero 2017, los trabajos de reacondicionamiento realizados por Petroamazonas EP en el campo CBS (1) del Oriente ecuatoriano, tuvieron costos que variaron de 100.000,00 USD a 1'300.000,00 USD por cada trabajo. Uno de los principales rubros de estos montos corresponde al alquiler de la torre de reacondicionamiento, razón por la cual es importante para la empresa optimizar los tiempos de ejecución de las operaciones que forman parte de este proceso.

\section{METODOLOGÍA}

El estudio partió de la recopilación de información de los reacondicionamientos realizados por la empresa Petroamazonas EP en el campo CBS desde enero 2015 a enero 2017. La fuente de información principal fueron los documentos y reportes oficiales de las áreas operativas de la empresa Petroamazonas EP que se preparan tanto en las oficinas centrales, como en el campo.

El reacondicionamiento de pozos es programado por las compañías operadoras, ejecutado por las compañías de servicios y supervisado por el área de operaciones de la empresa operadora y los organismos de control hidrocarburífero. Dada la importancia de este proceso, las compañías operadoras mantienen una base de datos por pozo que incluyen documentos como: solicitud de aprobación del trabajo de reacondicionamiento previo a su ejecución, programa de trabajo propuesto y reporte de resultados luego de realizado el trabajo.

Adicionalmente, el personal operativo del campo lleva un archivo en hoja de cálculo que incluye la descripción de actividades y el tiempo utilizado para cada una de las actividades del reacondicionamiento. Esta información se archiva por pozo, como parte del historial de producción y reacondicionamiento.

La información de todos los reacondicionamientos realizados en el período de estudio fue validada, descartándose datos incompletos 0 actividades esporádicas que no aportan al objetivo del estudio.

Para realizar la selección de los subprocesos candidatos a optimización, se agruparon las diferentes denominaciones que reciben dichas operaciones y sus respectivas actividades, en denominaciones estándar, a fin de poder compararlas y realizar el análisis estadístico. Una vez definidos los subprocesos estándar, se organizó la información disponible en hojas de cálculo, tabulando las operaciones con sus actividades principales.

Utilizando análisis de Pareto se determinaron las operaciones que tienen un nivel mayor de incidencia en el proceso general de reacondicionamiento y permiten realizar análisis estadísticos de mayor significancia por el número de datos disponibles.

Se determinaron los clientes internos y externos, tomando en cuenta que en este proceso intervienen, directa $\bigcirc$ indirectamente, personal de las áreas operativas, administrativas y de supervisión. La "voz del cliente" indicó que la característica clave que representa su satisfacción es el tiempo del proceso.

Utilizando el principio de que solo se puede mejorar lo que se puede medir (Pyzdek 2003), se analizaron las mediciones realizadas en los reacondicionamientos de pozos del campo CBS utilizando herramientas de control estadístico de procesos como son las gráficas de control.

Con el fin de determinar tendencias en las gráficas de control se ordenaron los eventos en forma temporal. En el análisis se determina si la gráfica de la serie de tiempo presenta patrones especiales como derivas, ciclos, repeticiones, aplanamientos, y se procedió a determinar los puntos fuera de rango.

En este estudio se utilizó como límites de control de los subprocesos, una desviación estándar a partir de la media de la población. Para determinación de fallas o comportamientos fuera de rango, se tomó en cuenta únicamente el límite superior. 
En cada uno de los subprocesos, se seleccionó el punto fuera de rango más alejado del comportamiento esperado, con el objetivo de realizar un análisis causa-efecto, que permita determinar las razones principales del comportamiento anómalo.

Tomando en cuenta el análisis realizado, se preparó una propuesta de mejoramiento, considerando la orientación al mejoramiento continuo y el compromiso con la calidad.

\section{RESULTADOS Y DISCUSIÓN}

En el período analizado de enero 2015 a enero 2017, Petroamazonas EP realizó 90 trabajos de reacondicionamiento en el campo CBS, de los cuales 61 corresponden a pozos con sistema de levantamiento con bomba eléctrica BES, tal como se muestra en la Figura 1.

De esta información se determinó que el enfoque del análisis debe orientarse a los trabajos de reacondicionamiento realizados en pozos que tienen el sistema de levantamiento tipo BES.

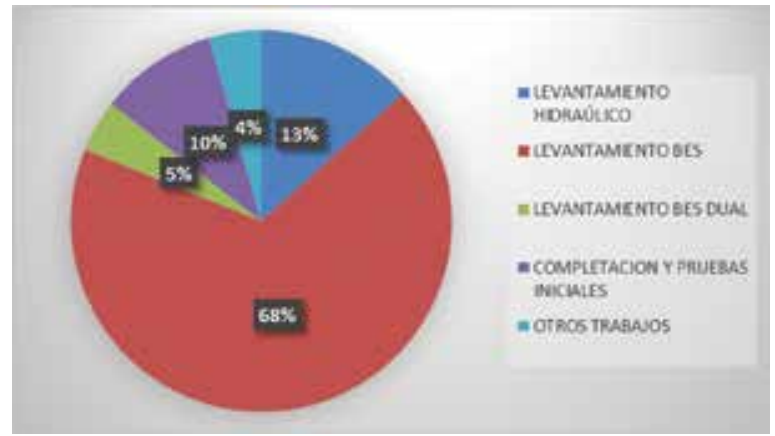

Figura 1. Trabajos de reacondicionamiento realizados en el periodo de análisis

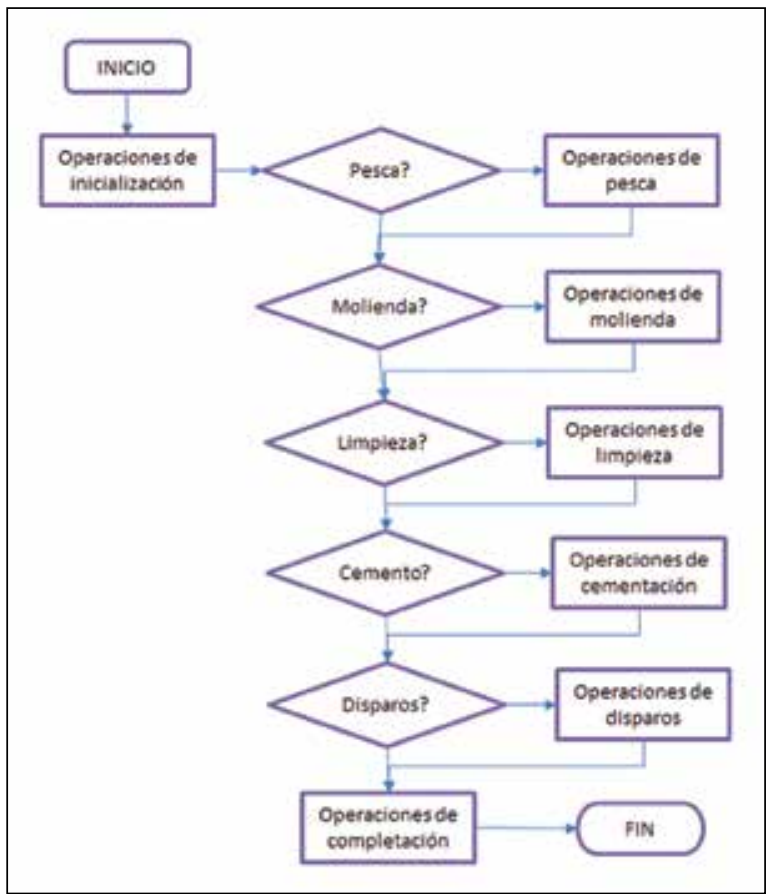

Figura 2. Diagrama de flujo de reacondicionamiento de pozos aplicable a las operaciones de la empresa Petroamazonas EP
En la Figura 2, se muestra un diagrama de flujo típico de un proceso de reacondicionamiento de pozos. Esta secuencia no es la única en las operaciones de reacondicionamiento, pero es la más común.

Los tiempos totales del proceso de reacondicionamiento dependen de las operaciones que se ejecuten. Con esta consideración, el análisis estadístico de tiempos debe orientarse a analizar cada uno de los subprocesos por separado.

En la Figura 3, se presenta la estadística de los trabajos realizados en el período de estudio por tipo de operación, en pozos con levantamiento BES.

Las estadísticas precedentes indican que el análisis debe enfocarse en las operaciones de inicialización, disparos, completación y finalización, las cuales representan, en total, el 78\% de las operaciones realizadas. De estas se seleccionaron las operaciones de inicialización y de completación para un análisis mas profundo.

\section{Análisis de Operaciones}

\section{a) Operaciones de Inicialización}

En la Figura 4, se indica la gráfica de control para operaciones de inicialización. Se toma como "no conformidad" cuando el valor observado sobrepasa el límite superior. Definido el límite superior de control

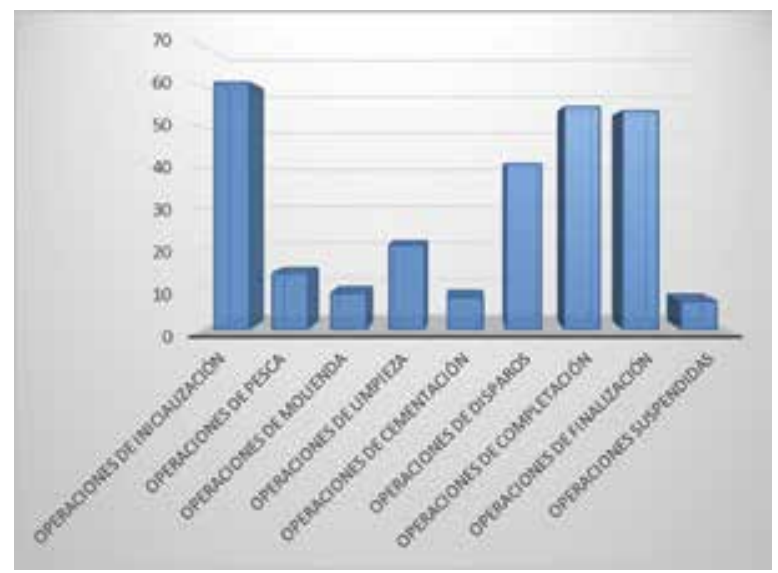

Figura 3. Operaciones realizadas en pozos con levantamiento BES en el periodo enero 2015 a enero 2017 por tipo de operación

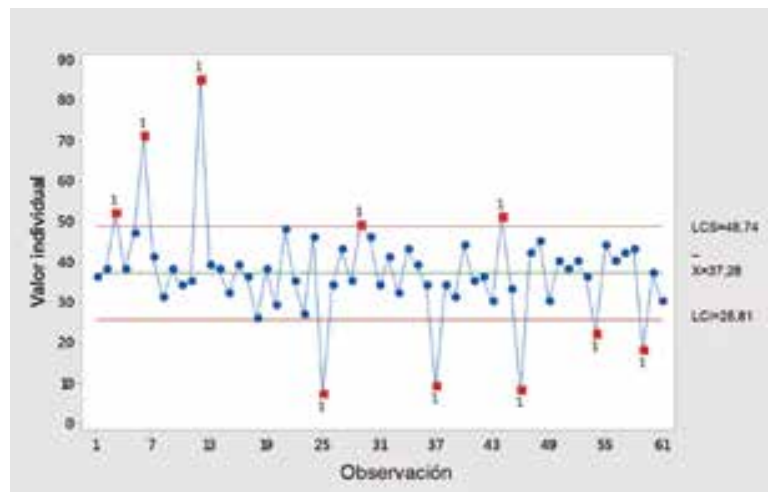

Figura 4. Gráfica de control para operaciones de inicialización 
a una desviación estándar a partir de la media, cinco puntos (5 puntos) están fuera del límite establecido.

La Figura 5, muestra el diagrama de Pareto de los tiempos promedios correspondientes a las actividades de Inicialización.

El diagrama muestra que la actividad de mayor consumo de tiempo y a la cual se debe poner mayor atención es sacar la BES del pozo.

En la gráfica de control para operaciones de inicialización se seleccionó el evento que está más alejado de la media, para su análisis. El problema se da en la actividad de recuperación de la BES.

Se realizó un diagrama causa-efecto en el que se describen las causas probables de la demora. En base a la revisión de la información histórica se determinó que la causa de la demora en el proceso, fue que se presentó una rotura de tubería que obligó a realizar trabajos adicionales como acondicionamiento de pescado, molienda y bajada de varios BHA de pesca hasta poder recuperar la BES. El diagrama causaefecto para demora en la recuperación de la BES se muestra en la Figura 6.

La recomendación en este caso para evitar que se produzca un evento similar el cual implica incremento en tiempo y uso de recursos, es que se debe utilizar tubería de producción nueva tipo A, de acuerdo a la denominación en la industria petrolera, o como alternativa utilizar tubería tipo B, revisada adecuadamente siguiendo la normativa respectiva.

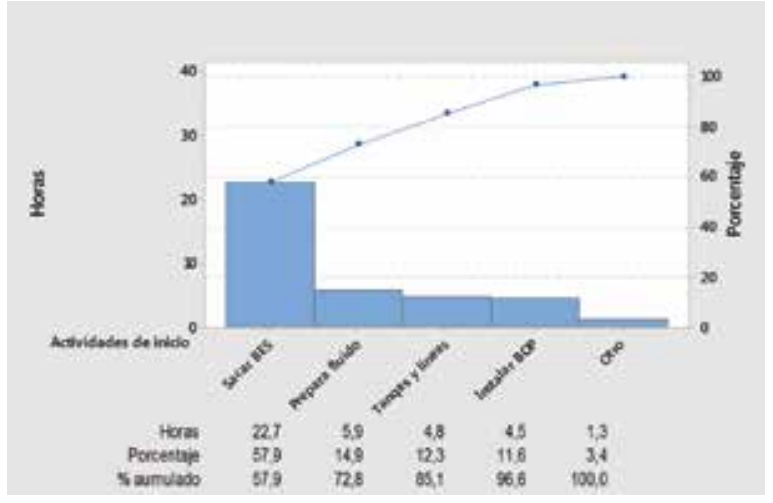

Figura 5. Diagrama de Pareto para actividades de inicialización

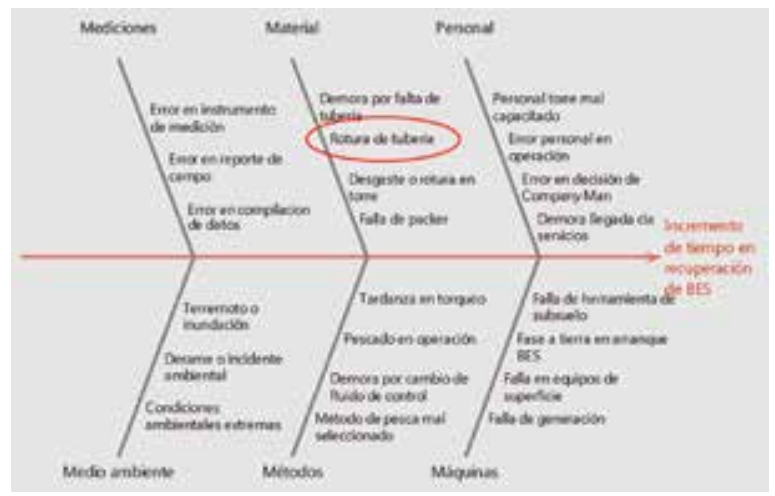

Figura 6. Diagrama causa-efecto para falla en recuperación de BES

\section{b) Operaciones de Completación}

En la Figura 7, se indica la gráfica de control para operaciones de completación, tomando como límites superior e inferior una desviación estándar a partir de la media. Ocho puntos están fuera del límite superior establecido.

La Figura 8, muestra el diagrama de Pareto de los tiempos promedios correspondientes a las actividades de completación.

El diagrama muestra que la actividad de mayor consumo de tiempo y a la que se debe poner mayor atención es la bajada del equipo BES tubo a tubo.

En la gráfica de control para operaciones de completación se han detectado ocho puntos (8 puntos) fuera del límite superior de los cuales se ha seleccionado el que está más alejado para su análisis. Este punto corresponde a un reacondicionamiento realizado en el mes de mayo del 2015.

Se ha preparado un diagrama causa-efecto en el cual se han descrito una serie de posibles causas de la demora. En base a la revisión de la información histórica se determina que la causa de la demora en la operación de completación fue la utilización de una herramienta de torqueo con llave computarizada. El diagrama causa-efecto para demora en la bajada del equipo BES se muestra en la Figura 9.

El torqueo con llave computarizada se da en los casos en que la tubería, debido a su aleación o delicadeza de los acoples, requiere de un torque

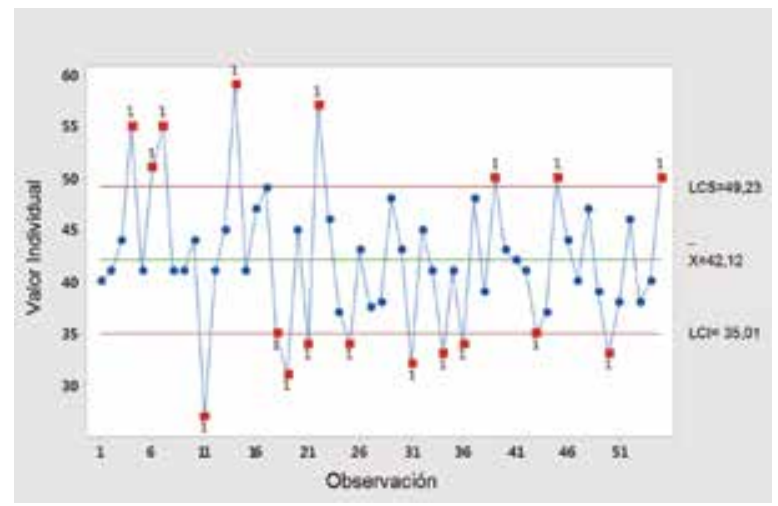

Figura 7. Gráfica de control para operaciones de completación

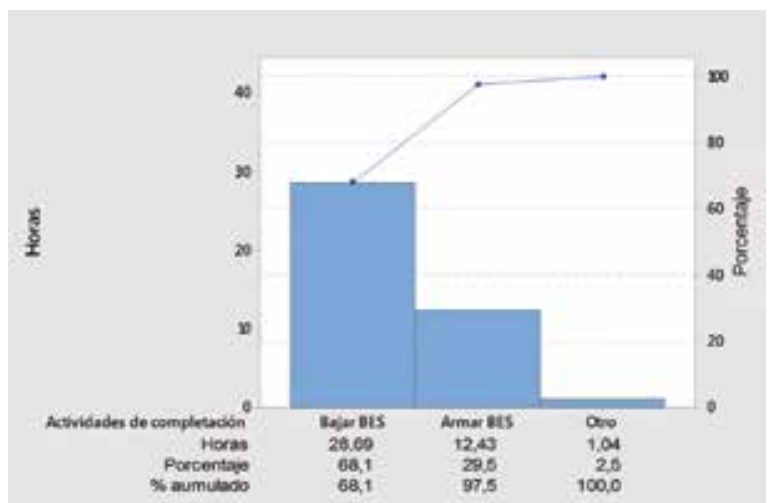

Figura 8. Diagrama de Pareto para actividades de completación 


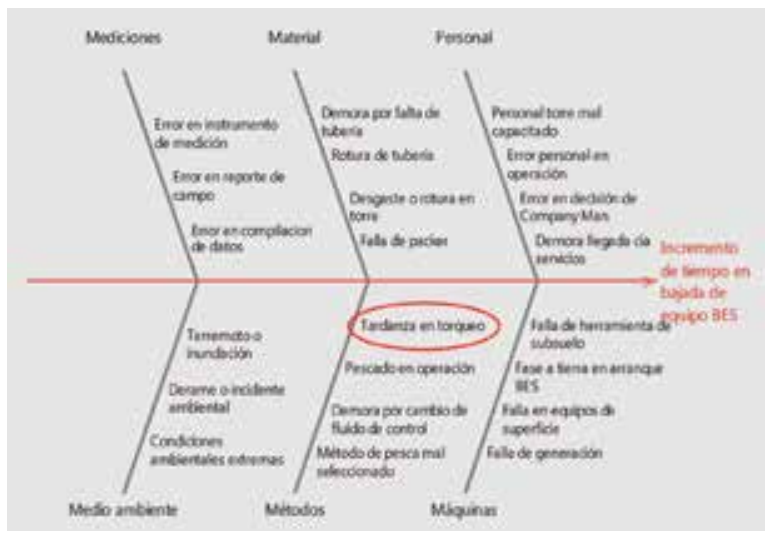

Figura 9. Diagrama causa-efecto para falla en bajada de BES

muy preciso al momento de realizar el acople tubo a tubo. La recomendación para esta situación es exigir una mayor pericia del personal en el acople computarizado para mejorar los tiempos.

\section{Propuesta de Mejoramiento y Control}

a) Consideraciones Generales

Las operaciones de reacondicionamiento de pozos es uno de los procesos más importantes en el conjunto de los procesos técnico-operativos de las empresas de producción y exploración petrolera.

En los trabajos de reacondicionamiento es fundamental tener visión de mediano y largo plazo, lo cual significa que se deben realizar las actividades planificadas, con una filosofía de calidad que disminuya la posibilidad de caer en problemas posteriores relacionados con una operación inicial deficiente.

En la implementación de un proyecto de mejora es necesario tomar en cuenta los siguientes factores de éxito: liderazgo, comunicación, reconocimiento, conocimiento y sostenimiento del esfuerzo emprendido.

Liderazgo: el programa debe estar respaldado por la Gerencia General de la empresa y los líderes del proyecto deben ser seleccionados en base a su calificación, comprometimiento y motivación.

Comunicación: Debe ser directa, oportuna y sincera. Solo un proceso transparente permitirá alcanzar los resultados esperados. Si se presentan problemas corresponde analizar sin tapujos a fin de determinar las causas y realizar los correctivos necesarios.

Reconocimiento: Se debe reconocer en primer lugar el alcance y superación de metas planteadas, pero también el esfuerzo realizado para alcanzar los objetivos.

Conocimiento: Es necesario que los participantes en el proceso conozcan las experiencias y camino ya recorrido en otras empresas y tomen contacto con los expertos en el tema, pues todo ello generará motivación y el convencimiento de que se sigue el camino correcto.

Sostenimiento del esfuerzo emprendido: Se debe tener presente que el camino del mejoramiento no es fácil, que no es una carrera de distancia corta, sino una de largo aliento. El esfuerzo debe mantenerse y superar las dificultades para alcanzar la meta de generar una filosofía de calidad.

\section{b) Plan de Mejoramiento}

El programa de mejoramiento tiene tres ejes: Capacitación, compromiso e involucramiento.

Capacitación: El reacondicionamiento de pozos es generalmente tercerizado: la torre de reacondicionamiento y los servicios relacionados son contratados con empresas de servicios petroleros. Por ello, se debe realizar un proceso de capacitación tanto al personal de la compañía operadora como de las compañías contratistas con el fin de que todos los involucrados tengan la misma visión del proceso.

Compromiso: Es imprescindible generar un sentimiento de compromiso con la calidad, tanto en el personal operativo como personal de apoyo. Este compromiso pasa por el concepto de hacerlo bien desde el inicio y con la mirada en el mediano y largo plazo.

Involucramiento: El involucramiento de los niveles gerenciales, al igual que el de los niveles operativos, es fundamental para dar al programa un carácter oficial, de tal manera que todos los involucrados vean al proceso de mejoramiento, como un objetivo estratégico empresarial.

\section{c) Programa de Control}

El programa de control se basa en dos ejes principales: Realizar y reportar mediciones de tiempo más detalladas en los procesos petroleros e incorporar los sistemas de control estadístico que se utilizan en otras industrias para detectar fallas y recomendar correcciones. El programa de control propuesto se muestra en la Tabla 1.

Se debe incrementar y reportar de manera oficial mediciones de tiempo más detalladas, sobre todo en las actividades de mayor consumo de tiempo, como son la bajada de BES tubo a tubo, recuperación de BES quebrando y pruebas de producción. Para ello es necesario subdividir estas actividades en etapas que tengan una duración menor.

Como parte de las actividades del campo a más de llevar el control por curvas de tiempo, se debe llevar un sistema de gráficas de control, que permita identificar las actividades que están fuera del estándar. Adicionalmente, los ingenieros de campo tienen que aplicar el análisis causa-efecto, a fin de determinar los motivos por los cuales no se tuvo el desempeño esperado y en base a ello tomar los correctivos necesarios para que se minimice la posibilidad de que vuelva a suceder el evento no deseado.

\section{CONCLUSIONES}

- El análisis de los requerimientos de los clientes internos y externos del proceso de reacondicionamiento de pozos, determinó que el parámetro clave que satisface la voz del cliente es el tiempo. 
Tabla 1. Programa de control para reacondicionamiento en pozos BES

\begin{tabular}{|c|c|c|}
\hline \multicolumn{3}{|c|}{ PROGRAMA DE CONTROL } \\
\hline Tipo de control & Control & Frecuencia \\
\hline $\begin{array}{l}\text { Controles } \\
\text { Generales: } \\
\text { mantener } \\
\text { los controles } \\
\text { actuales }\end{array}$ & $\begin{array}{l}\text { Datos generales del } \\
\text { trabajo } \\
\text { Sumario de } \\
\text { resultados } \\
\text { Diagrama después } \\
\text { del trabajo } \\
\text { Hoja de tiempo del } \\
\text { trabajo }\end{array}$ & $\begin{array}{l}\text { Para cada trabajo } \\
\text { de WO }\end{array}$ \\
\hline $\begin{array}{l}\text { Controles } \\
\text { de tiempo } \\
\text { adicionales }\end{array}$ & $\begin{array}{l}\text { Actividades de } \\
\text { alta demanda de } \\
\text { tiempo como bajada } \\
\text { y subida de BHA, } \\
\text { controlada por } \\
\text { profundidad: cada } \\
1000 \text { pies }\end{array}$ & $\begin{array}{l}\text { Para cada } \\
\text { actividad }\end{array}$ \\
\hline $\begin{array}{l}\text { Determinación } \\
\text { de operaciones } \\
\text { fuera de rango }\end{array}$ & $\begin{array}{l}\text { Preparación de } \\
\text { gráficas de control } \\
\text { por operación }\end{array}$ & $\begin{array}{l}\text { Para cada } \\
\text { Operación } \\
\text { considerando } \\
\text { todos los trabajos } \\
\text { del periodo }\end{array}$ \\
\hline $\begin{array}{l}\text { Análisis de causa } \\
\text { raíz durante el } \\
\text { proceso }\end{array}$ & $\begin{array}{l}\text { Preparación del } \\
\text { diagrama de espina } \\
\text { de pescado } \\
\text { Determinación de } \\
\text { causa raíz }\end{array}$ & $\begin{array}{l}\text { Para cada } \\
\text { Operación } \\
\text { fuera de rango } \\
\text { considerando } \\
\text { todos los trabajos } \\
\text { del periodo }\end{array}$ \\
\hline $\begin{array}{l}\text { Estadística } \\
\text { transversal de } \\
\text { falla de equipo } \\
\text { de superficie y } \\
\text { fondo: análisis de } \\
\text { Pareto }\end{array}$ & $\begin{array}{l}\text { Tubería } \\
\text { Packers } \\
\text { Fluido de control } \\
\text { Cable } \\
\text { Generadores } \\
\text { Otros }\end{array}$ & $\begin{array}{l}\text { Para todos } \\
\text { los trabajos } \\
\text { del periodo, } \\
\text { considerando } \\
\text { proveedor y } \\
\text { precios }\end{array}$ \\
\hline
\end{tabular}

- El análisis de Pareto aplicado a las operaciones de reacondicionamiento realizadas por Petroamazonas EP en el campo CBS desde enero 2015 a enero 2017, determinó que entre las principales operaciones a ser consideradas en un proceso de mejoramiento están las de inicialización y completación.

- Las gráficas de control indicaron que en las operaciones de inicialización, cinco de los 61 puntos están fuera de rango. En las operaciones de Completación 8 de los 55 puntos están fuera de rango.

- El análisis causa-efecto realizado a las diferentes operaciones, determinó que las principales causas de falla se deben a mala calidad del material utilizado, falla de equipos de subsuelo y superficie y falta de pericia del personal en operaciones no repetitivas como las de torqueo.

- El estudio indicó la necesidad de aumentar los controles de tiempo en actividades de gran consumo de ese recurso a fin de contar con los datos que permitan determinar acciones de corrección y mejora.

- El programa de mejoramiento planteado permitirá optimizar el proceso de reacondicionamiento de pozos, reduciendo el número de eventos fuera de rango, generando una filosofía de mejoramiento continuo y un compromiso con la calidad.

\section{REFERENCIAS}

- Deming, W. E. (1989). Calidad, productividad y competitividad: La salida de la crisis. (J. Nicolau, Trad.) Madrid, España: Ediciones Díaz de Santos S. A.

- Knowles, G. (2011). Six Sigma. Recuperado el Enero de 2017, de Bookboon.com: ISBN-97887-7681-852-4.

- OPEC. (2016). Annual Statistical Bulletin 2016. Vienna, Austria: Organization of the Petroleum Exporting Countries.

- Pyzdek, T. (2003). The Six Sigma Handbook, a Complete Guide for Green Belts, Black Belts, and Managers at All Levels. United States of America: McGraw-Hill.

- Ruiz, R (2017). Propuesta de mejoramiento del proceso de reacondicionamiento de pozos en un campo del Oriente Ecuatoriano aplicando la metodología Seis Sigma. EPN, Quito, Septiembre 2017. 